\title{
A comparison of 11-year mesospheric and lower thermospheric winds determined by meteor and MF radar at $69^{\circ} \mathrm{N}$
}

\author{
Sven Wilhelm, Gunter Stober, and Jorge L. Chau \\ Leibniz Institute of Atmospheric Physics at the University of Rostock, Kühlungsborn, Germany \\ Correspondence to: Sven Wilhelm (wilhelm@iap-kborn.de)
}

Received: 13 February 2017 - Revised: 6 July 2017 - Accepted: 7 July 2017 - Published: 31 July 2017

\begin{abstract}
The Andenes Meteor Radar (MR) and the Saura Medium Frequency (MF) Radar are located in northern Norway $\left(69^{\circ} \mathrm{N}, 16^{\circ} \mathrm{E}\right)$ and operate continuously to provide wind measurements of the mesosphere and lower thermosphere (MLT) region. We compare the two systems to find potential biases between the radars and combine the data from both systems to enhance altitudinal coverage between 60 and $110 \mathrm{~km}$. The systems have altitudinal overlap between 78 and $100 \mathrm{~km}$ at which we compare winds and tides on the basis of hourly winds with $2 \mathrm{~km}$ altitude bins. Our results indicate reasonable agreement for the zonal and meridional wind components between 78 and $92 \mathrm{~km}$. An exception to this is the altitude range below $84 \mathrm{~km}$ during the summer, at which the correlation decreases. We also compare semidiurnal and diurnal tides according to their amplitudes and phases with good agreement below $90 \mathrm{~km}$ for the diurnal and below $96 \mathrm{~km}$ for the semidiurnal tides.

Based on these findings we have taken the MR data as a reference. By comparing the MF and MR winds within the overlapping region, we have empirically estimated correction factors to be applied to the MF winds. Existing gaps in that data set will be filled with weighted MF data. This weighting is done due to underestimated wind values of the MF compared to the MR, and the resulting correction factors fit to a polynomial function of second degree within the overlapping area. We are therefore able to construct a consistent and homogenous wind from approximately 60 to $110 \mathrm{~km}$.
\end{abstract}

Keywords. Radio science (instruments and techniques)

\section{Introduction}

During the past decades, radars have been used to investigate mesospheric phenomena, e.g., polar mesospheric echoes
(Rapp et al., 2008, Suzuki et al., 2013, Chau et al., 2014) and atmospheric dynamics (e.g. Andrews et al., 1987, Fritts et al., 2012, Iimura et al., 2015). Mesospheric radars are distributed over the whole globe and depending on their antenna arrays, frequencies, locations, and transmitting power, they provide valuable information about winds at different vertical and spatial scales. One of the main advantages of radar systems, compared to other remote sensing techniques for the mesosphere and lower thermosphere (MLT), is that they provide continuous measurements independent of weather conditions. A crucial aspect of the measured winds is the reliability of each technique. This requires a proper understanding of the underlying scattering processes and possible instrumental effects, analysis related simplifications, and assumptions that could introduce biases or systematic errors in the derived winds (Reid, 2015).

In this study we analyze data from the Saura Medium Frequency (MF) Radar and the Andenes Meteor Radar (MR), which are both located on the island of Andøya in northern Norway $\left(69^{\circ} \mathrm{N}, 16^{\circ} \mathrm{E}\right)$. The comparison is done based on data collected between 2004 and 2014. This study pursues two primary goals. First we want to quantify potential biases between the two techniques, and secondly we intend to merge both wind fields in order to compile a consistent and homogenous wind from approximately 60 to $110 \mathrm{~km}$ of altitude. Further we examine whether it is possible to fill gaps in the time series and generate a long continuous time series, ideally throughout the whole comparison period, that is suitable to study atmospheric patterns with periods between months and years. Similar comparisons have already been performed within the past few decades by Valentic et al. (1997), Hocking et al. (2001b), and Hall et al. (2005) with smaller data sets and different locations. Their results showed particularly good agreement between MF and MR for alti- 
tudes between 75 and $85 \mathrm{~km}$, but they indicate larger discrepancies at higher altitudes in the obtained winds.

Former studies very often used MF winds obtained by full correlation analysis (FCA). Most MFs employ a wide beam and use only three receiving antennas. However, due to the large observation volume there were issues with the analysis that can mainly be attributed to the underlying assumption that the FCA technique requires homogeneous volumefilled backscattering within the beam volume (Sommer et al., 2016). A technical upgrade in 2003 for the Saura MF radar allows us to operate the MF in a Doppler beam swinging (DBS) mode and to derive winds from multiple oblique beams (Singer et al., 2008). The main difference of Saura, compared to many other MF radars, is its large antenna array (Mills Cross), which permits a rather narrow beam. The benefit of the MF DBS mode is that a wind and tidal comparison with MR can now be done based on the same wind fit routine. We operated the MF in DBS mode for this comparison.

The article is structured as follows. First we describe the radar systems used in this study. We explain the method to determine the wind and tides for both systems in Sect. 3, and in Sect. 4 we compare the winds and tides obtained from both systems. The discussion and conclusions are found in Sects. 5 and 6 , respectively.

\section{Experimental setup}

In this study we present observations from two different radars located at Andenes $\left(69.3^{\circ} \mathrm{N}, 16^{\circ} \mathrm{E}\right)$. The systems are the Andenes Meteor Radar, which measures radial velocities from meteor trails, and the Saura MF radar, which obtains measurements from refraction index variations due to dynamic processes (e.g., gravity waves) and D-layer ionization and associated irregularities (Reid, 2015). The technical details for both systems are summarized in Table 1 .

In 2001 the Andenes Meteor Radar started its continuous operation up to the present. The radar has been updated several times and the peak transmitting power has increased from 6 to $30 \mathrm{~kW}$. The radar consists of one circular polarized transmitting 3-element Yagi antenna and five circular polarized receiving 2-element Yagi antennas. The receiver antenna array is arranged as five-antenna Jones configuration (Jones et al., 1998). The system operates at 32.5 MHz. Most specular meteors are detected at an approximate altitude of $90 \mathrm{~km}$ (Stober and Chau, 2015, Vierinen et al., 2016). At this altitude, the observed measurement volume has a diameter of $\sim 400 \mathrm{~km}$. A detailed description of the so-called All-Sky meteor radar is found in Hocking et al. (2001a).

The Saura HF radar, historically called "MF", is located $15 \mathrm{~km}$ south of the Andenes MR location and operates on a frequency of $3.17 \mathrm{MHz}$. Although the frequency used is in the HF band, Saura was designed and built as an MF radar (Singer et al., 2003). In 2002 the Saura MF started its observations. The transmitting and receiving antenna is formed by 29 crossed half-wave dipole antennas in a Mills Cross arrangement. In addition to differential absorption and phase measurements of electron density, the system is able to provide winds (Singer et al., 2003) based on atmospheric irregularities (Briggs, 1984). The altitudinal coverage ranges from 50 to $100 \mathrm{~km}$. Since 2003, the radar has allowed for Doppler beam swinging (DBS) experiments, which were done for this study, and spaced antenna applications. In DBS mode, offzenith beams towards N, S, E, W and NW, NE, SW, SE at zenith angles between $6.8^{\circ}$ and $7.3^{\circ}$ were used. More technical information for the Saura MF radar can be found in Singer et al. (2003), Singer et al. (2008).

\section{Wind and tidal analysis}

We compared wind measurements obtained from the MR and MF instruments using a DBS retrieval technique. In the case of the MR, horizontal winds are preserved using a modified All-Sky-fit Doppler approach (Hocking and Thayaparan, 1997, Hocking et al., 2001a, Stober et al., 2017), for which an ensemble of at least five randomly distributed meteors in a given time and altitude bin are used to estimate the 3-D wind. In the case of the Saura MF, winds are derived by combining the radial velocity measurements from four oblique and one vertical beam. In both cases, the wind vector $(u, v, w)$ is obtained from the following set of equations:

$v_{i}(\theta, \phi)_{\mathrm{rad}}=u \cos \phi_{i} \sin \theta_{i}+v \sin \phi_{i} \sin \theta_{i}+w \cos \theta_{i}$,

where $u, v$, and $w$ are the zonal, meridional, and vertical wind components, $\theta_{i}$ and $\phi_{i}$ are the zenith and the azimuth angle, respectively, and $v_{\text {rad } i}$ is the radial velocity for each measurement. Hourly winds are obtained by binning the data in height and time. We use a $2 \mathrm{~h}$ sliding window centered at the reference time. A similar procedure is used for the altitude bins by applying a $3 \mathrm{~km}$ window shifted by $2 \mathrm{~km}$ and centered at a reference altitude.

The wind is computed considering the statistical uncertainties in each radial velocity measurement by applying an additional Gaussian weighting depending on its time of occurrence with respect to the reference time and for the altitude coordinate. The Gaussian-shaped window is used to provide an additional weighting of the individual meteors within a time and altitude bin. The regularization already estimates a temporal or vertical shear. This shear is used to penalize the impact of each measured radial velocity depending on its temporal or spatial offset from the reference grid. Further, this shear provides an estimate of the shear error for each time and altitude. This error is added to the statistical uncertainty in the radial velocity measurement. More information about the applied regularization can be found in Stober et al. (2017).

The accuracy of the wind is obtained from the fitting procedure to estimate the wind by taking into account the number of measurements per bin and the statistical uncer- 
Table 1. Technical data and main parameters for the radars used in this study.

\begin{tabular}{lll}
\hline & Meteor radar & Medium-frequency radar \\
\hline Location & $69.3^{\circ} \mathrm{N}, 16^{\circ} \mathrm{E}$ & $69.3^{\circ} \mathrm{N}, 16^{\circ} \mathrm{E}$ \\
Scattering processes & meteor trail & irregularities \\
Wind analysis height range & $75-110 \mathrm{~km}$ & $50-100 \mathrm{~km}$ \\
Wind analysis vertical resolution & $2 \mathrm{~km}$ & $2 \mathrm{~km}$ \\
Antenna & crossed & crossed \\
Frequency & $32.55 \mathrm{MHz}$ & $3.17 \mathrm{MHz}$ \\
Power & $30 \mathrm{~kW}$ & $116 \mathrm{~kW}$ \\
\hline
\end{tabular}

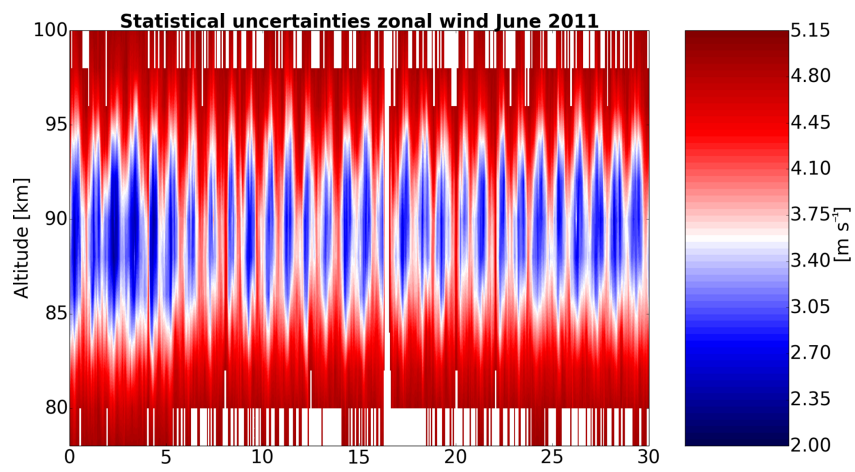

Figure 1. Statistical uncertainties for the hourly zonal wind of the MR for June 2011.

tainties in the measurements in the error covariance matrix. This leads to uncertainties of approximately $2-6 \mathrm{~m} \mathrm{~s}^{-1}$ for the hourly MR winds with larger errors at the edges of the observation range. This can be seen in Fig. 1, which shows the MR uncertainties based on the hourly zonal wind values for June 2011. The same structure occurs for the meridional wind component (not shown here). A more detailed description of the fitting routine can be found in Stober et al. (2017).

Furthermore, we apply two additional assumptions to simplify the retrieval method. These assumptions are (1) zero acceleration within the altitude and time bin and (2) zero vertical velocity, which leads to a smoother wind field solution. The assumptions are used to constrain our wind retrieval by applying Tikhonov regularization (Aster, 2013, Stober et al., 2017). Considering the rather large observation volume of a meteor radar, which has a diameter of approximately $400 \mathrm{~km}$ at $90 \mathrm{~km}$ of altitude, it is not advisable to fit for the vertical velocity directly. A wind field spanning such a volume likely also shows patterns of horizontal divergence or convergence. Using a simple gradient expansion of the wind field (Browning and Wexler, 1968 or Waldteufel and Corbin, 1978) shows that the horizontal divergence and the vertical wind are linked. Thus, applying the standard 3-D wind fit as it is typically applied for MST radars (Hooper et al., 2007) is not applicable to MRs.

The Saura MF winds are obtained from the radial velocity measurements. The determination of winds for the medium-frequency radar is done with the same wind fit routine based on the capability of using our five-beam experiments with one vertical and four oblique. In DBS mode, off-zenith beams towards N, S, E, W and NW, NE, SW, SE at zenith angles between 6.8 and $7.3^{\circ}$ were formed for the measurements. The radial velocities of the MF radar are estimated using the momentum method (Strauch et al., 1984), and there is no available information about the statistical uncertainties in the radial velocity measurements (Hooper et al., 2007). Hence, we are not able to conduct a full error propagation to estimate the statistical uncertainties in the observed winds.

Theoretically, it would be possible to compare the radial velocities of meteors, which occur directly in the Saura beam, while Saura is measuring the radial Doppler. This would be more direct than using meteor wind fits. However, the systems are located only $20 \mathrm{~km}$ apart, and the Saura beam only points at approximately $6.8^{\circ}$ off-zenith, so the number of meteors would not be sufficient to provide statistically significant winds by the meteor radar. Hall et al. (2006) already showed that the number of detected meteors in zenith over and within an MF beam is strikingly marginal. Only by interpolating over a longer time bin would it be possible to provide winds by the meteor system, but these winds are not meaningful for our study.

The tidal components are obtained by estimating the diurnal, semidiurnal, and terdiurnal tidal oscillation components and applying an adaptive spectral filter (Stober et al., 2017). Therefore we decompose the time series as follows:

$$
u, v=u_{0}, v_{0}+\sum_{n=1}^{3} a_{n} \sin \left(2 \pi / T_{n} \cdot t\right)+b_{n} \cos \left(2 \pi / T_{n} \cdot t\right)
$$

Here $u_{0}$ and $v_{0}$ are the mean zonal and meridional wind. $T_{n}$ takes values of 24,12 , and 8 for the three tidal components and $a_{n}$ and $b_{n}$ are coefficients of the tidal amplitude and the associated phases for each wind component. In this study we focused on the diurnal and semidiurnal tidal amplitudes and phases using a 5-day mean centered at the respective day to suppress smaller-scale variations in the amplitudes. We apply the same procedure to both data sets. 


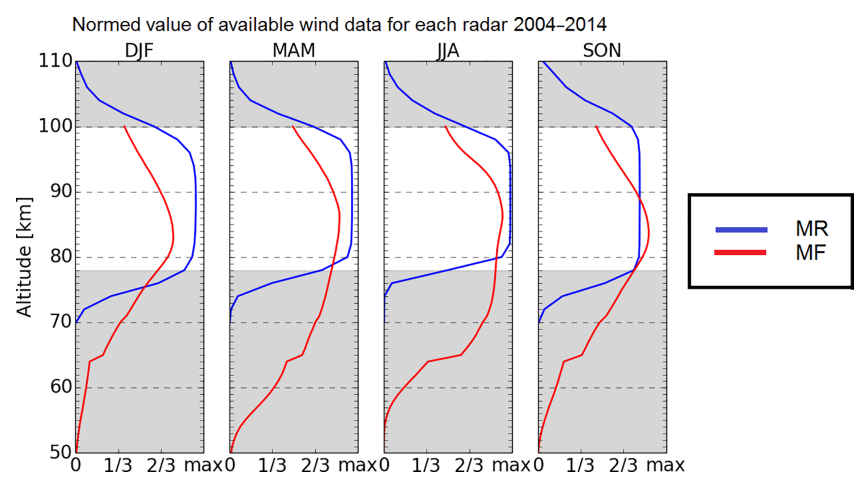

Figure 2. Altitude profile of the available wind data for the MR (blue) and the MF (red) according to season. The gray area shows the wind values for which at least one system is able to provide winds. The white area corresponds to the overlapping data area, which is used for comparison.

\section{Results}

\subsection{Wind comparison between radar systems}

In the following section we compare the obtained hourly winds. In Fig. 2 we show altitude profiles of the available wind data for both radars averaged over the seasons December-February (DJF), March-May (MAM), JuneAugust (JJA), and September-November (SON). The white shaded area indicates the overlapping altitude range used for the comparison.

The MR measurements clearly have the best statistics between 80 and $96 \mathrm{~km}$, as there is a clear maximum in the number of detections at these altitudes. In SON the amount of available wind data is reduced compared to the rest of the year due to maintenance in the years 2013 and 2014. Below $78 \mathrm{~km}$ and above $104 \mathrm{~km}$, the number of continuous MR wind observations is decreased for all seasons due to the decreased number of meteor detections. These reduced statistics are reflected by the statistical uncertainties, which are increased for these altitudes.

For the MF radar, the number of available wind values varies highly with background ionization, so during the summer the amount of valid wind data is the largest, while during the winter the amount is decreased. The measurements have the best statistics for all seasons between 80 and $88 \mathrm{~km}$, and within the white shaded area the number of valid winds decreases strongly above $92 \mathrm{~km}$ to $\sim 50 \%$ of the maximum possible number of wind measurements.

In order to assess whether there are systematic seasonal deviations between the two radar systems, we compile a yearly composite by using a 5-day mean centered at the respective day. This composite mainly suppresses the impact of short-term variations, e.g., tides and gravity waves. The composite for both wind components and both radar systems for the years 2004-2014 is shown in Fig. 3. The left two fig- ures show the typical mesospheric annual wind climatology for the zonal wind component with eastward-directed wind during the winter, a wind reversal during the spring, and a vertical wind shear in summer with eastward winds above $\sim 90 \mathrm{~km}$ and westward winds below $\sim 88 \mathrm{~km}$. This is consistent with the results presented in Hoffmann et al. (2010). The strongest zonal mean winds occurs during winter and summer with a mean wind velocity up to $\pm 40 \mathrm{~m} \mathrm{~s}^{-1}$. Certainly even with smoothing the typical planetary wave activity during the winter season is reflected by both radars. Between March and April the zonal wind component changes from eastward to westward over the whole observed altitude range. However, the gradient during the transition of the wind direction is stronger at all altitudes for the MR than for the MF winds. During the summer, both radars show a strong westward-directed wind with values of about $20 \mathrm{~m} \mathrm{~s}^{-1}$ below $85 \mathrm{~km}$ and eastward-directed winds above $90 \mathrm{~km}$. Nevertheless, there is a clear discrepancy in the magnitude above $90 \mathrm{~km}$. The MR measures values around $35 \mathrm{~m} \mathrm{~s}^{-1}$, whereas the MF shows lower values around $10 \mathrm{~ms}^{-1}$. The systematic underestimation of the MF winds compared to MR winds was pointed out by other studies; e.g., Valentic et al. (1997), Hocking and Thayaparan (1997), Hall et al. (2005) and Singer et al. (2008).

In addition to the amplitude of the wind, we show in Fig. 4 the comparison of the wind direction between the two radars for winter and summer at the altitudes of 80, 86, and $92 \mathrm{~km}$. These scatter plots are based on hourly zonal and meridional wind data and mainly show good agreement between the radars. With increasing altitudes, the compared azimuth angles of the systems start to diverge from the line of equality.

\subsection{Comparison of tidal signatures}

Now we proceed to analyze the MF and MR winds with respect to the tides. The tidal signatures should be almost identical considering the global structure and long vertical wavelengths of these waves. The upper part of Fig. 5 shows the 12 and $24 \mathrm{~h}$ tidal amplitude for a selected year (2011), and the lower part shows the differences between the MR and the MF tides for the zonal wind. We focus on a comparison of the zonal component because the differences in the winds are more dominant. The stronger tidal component at the MLT is the semidiurnal tide, which reaches mean values of approximately $\sim 40 \mathrm{~m} \mathrm{~s}^{-1}$, whereas the diurnal tide reaches values of $\sim 25 \mathrm{~m} \mathrm{~s}^{-1}$. This pattern with similar values also occurs in the other years. In the diurnal component a strong enhancement of the amplitude occurs during June and July below $85 \mathrm{~km}$ for both data sets. During September and October the systems measure an enhancement of the semidiurnal tide above $85 \mathrm{~km}$ in both tidal components with higher values for the MR. The main discrepancies between the two radar systems appear at higher altitudes; the amplitude of the MR increases with height, while the amplitude of the MF 


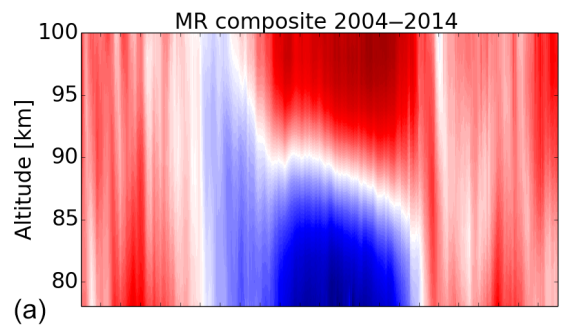

(b)
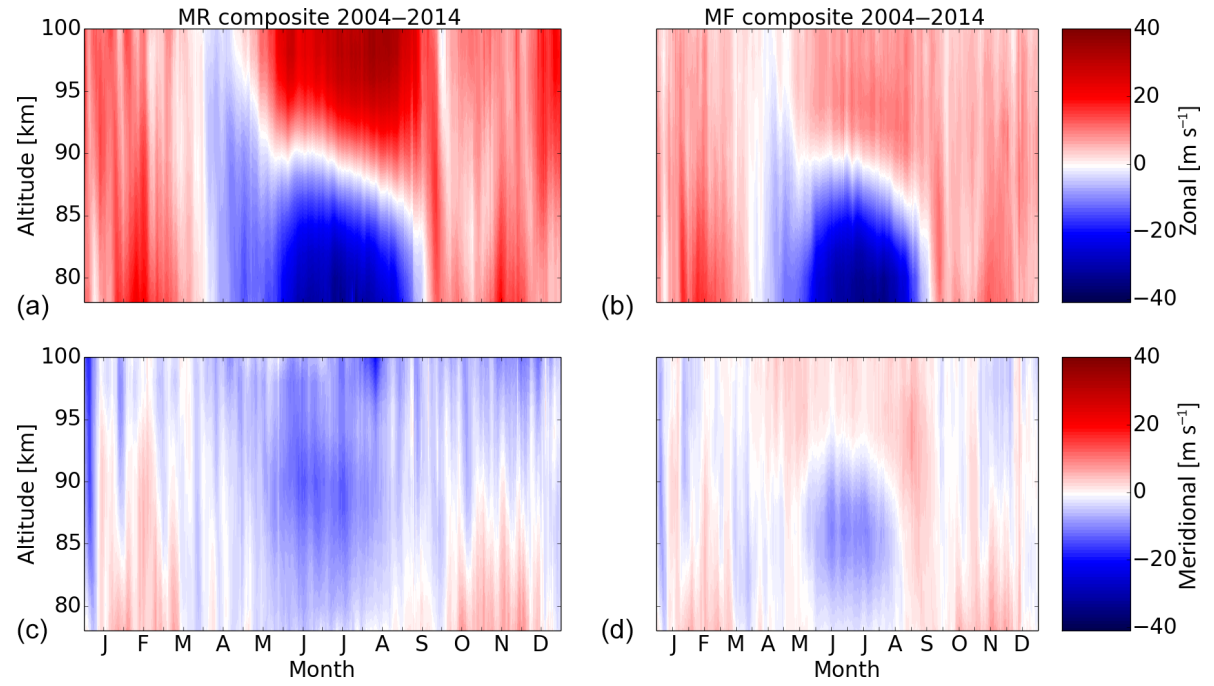

(d)

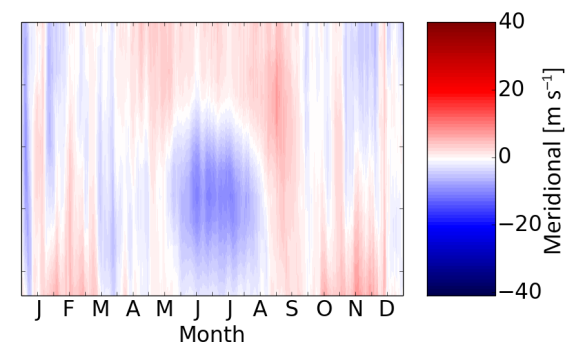

Figure 3. Composite of zonal $(\mathbf{a}, \mathbf{b})$ and meridional (c, d) wind component for meteor radar (a, c) and MF radar (b, d) for the years 2004-2014. The data have been smoothed by using a 5-day mean centered at the respective day.

(a)

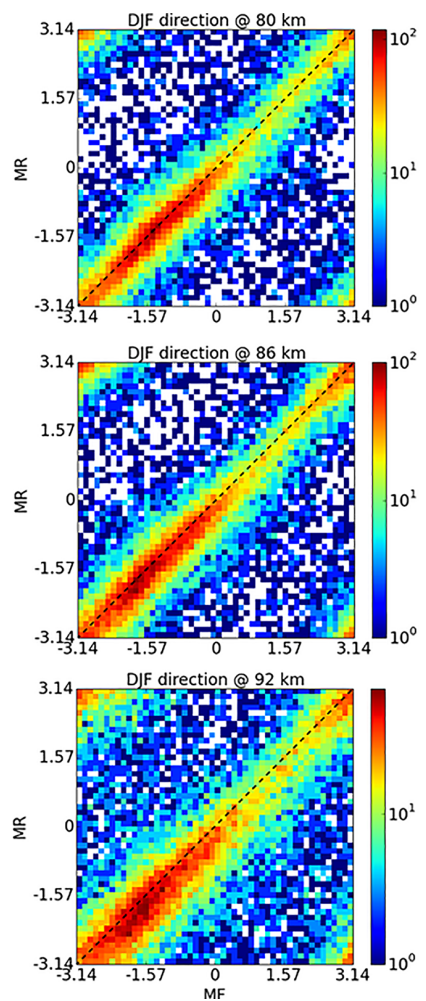

(b)
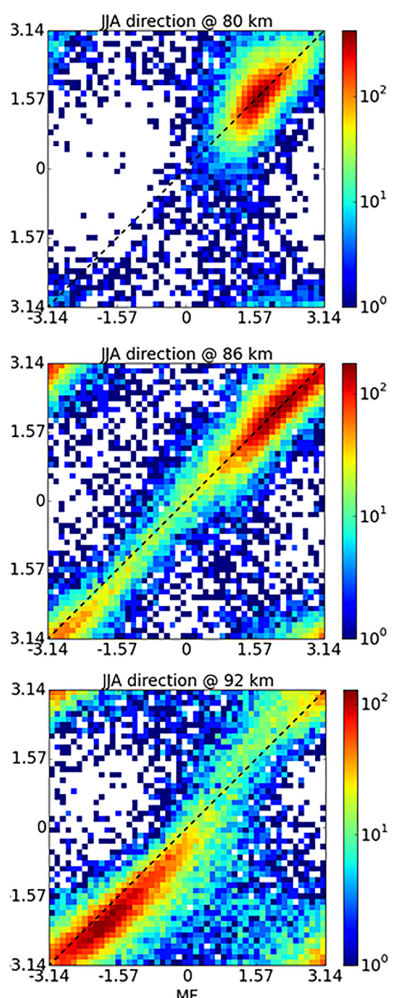

Figure 4. Scatter plots of the wind direction for DJF (a) and JJA (b) for 80,86 , and $92 \mathrm{~km}$. The black dashed line shows the line of equality.

stays nearly constant. Below $90 \mathrm{~km}$ the pattern and size of both systems look quite similar.

Within every frame in the lower part of Fig. 5, the differences in each component are shown for different seasons. Figure 5a shows that the semidiurnal tidal amplitudes from the MR are larger than the MF amplitude at nearly every altitude. The differences increase with increasing heights. This behavior is evident for every season of the year. Nevertheless, the differences at every altitude are within the given uncertainties, which reflects the seasonal variability. For the seasonal difference in the diurnal tidal component (right panel), the values of both radars are equal within the given uncertainties. With larger altitudes, the MR radar shows larger mean values than the MF radar. Similar behavior for the amplitude and the differences in each tidal component can be found in the meridional wind (not shown here).

In addition to the amplitudes we compare the phases of the tides (Fig. 6). The phases can reach values between $-\pi$ and $\pi$. If the phases for one system show a value of 0 and for the other system the value is $\pi$, then the phases of the two systems point in opposite directions, which corresponds, e.g., in the case of the diurnal tide, to a phase offset of $12 \mathrm{~h}$ between the two radars. Figure 6 shows the mean seasonal (winter and summer) zonal phase structure based on seasonal means for the winter and summer from 2004 to 2014. Figure $6 b$ shows that the semidiurnal phases of both radars for the winter months are equal between 78 and $94 \mathrm{~km}$ and similar up to $98 \mathrm{~km}$ within the variance. For the summer in the altitude range between 78 and $96 \mathrm{~km}$, the systems provide the same tidal mean phases within the variance. The given uncertainties show the statistical variability, which increases for both systems with height for the semidiurnal tide. The meridional semidiurnal component (not shown here) provides reasonable agreement between the two radars within the errors for every altitude. 

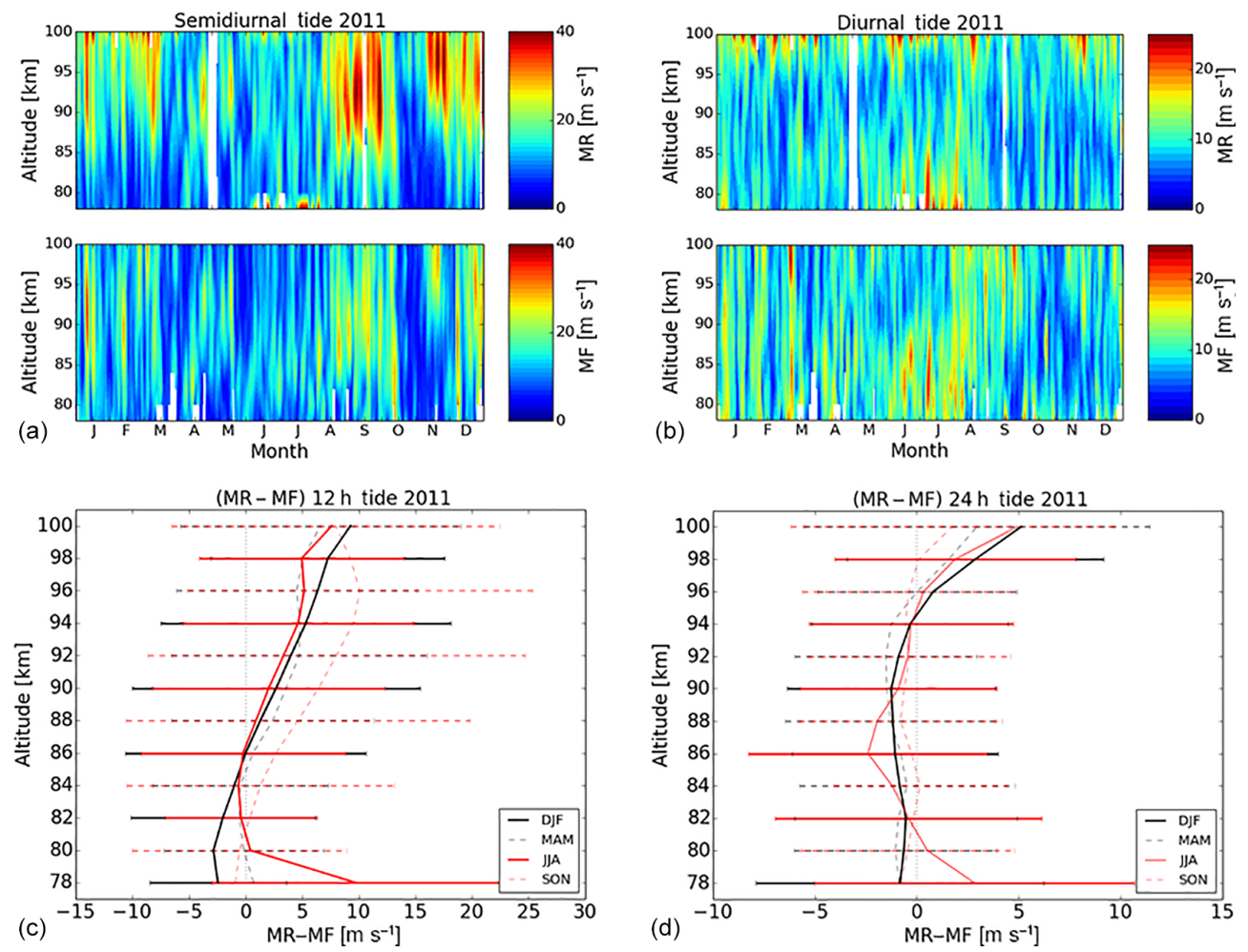

Figure 5. Semidiurnal (a) and diurnal tides (b) in 2011 for both systems. The white areas are missing values bases on outliers or due to maintenance. (c, d) Calculated difference for SDT and DT between MR and MF for the seasons DJF (solid black), MAM (dashed black), JJA (solid red), and SON (dashed red) for the year 2011. The error bars correspond to the seasonal variability.
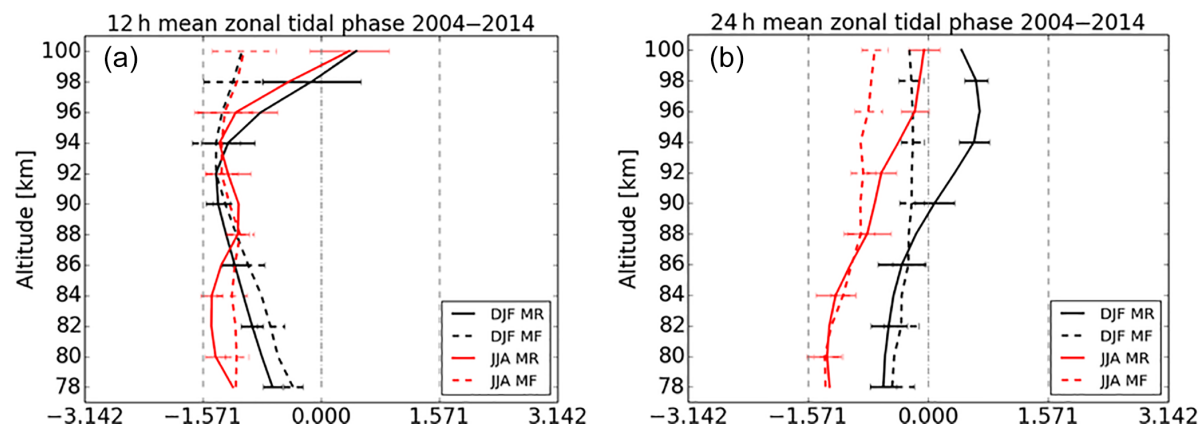

Figure 6. Vertical mean phase structure for the summer and winter seasons between 2004 and 2014. Semidiurnal (a) and diurnal tidal phases (b) of the zonal wind component for winter (DJF; in black) and summer (JJA; in red) for the meteor radar (solid) and the medium-frequency radar (dashed). The error bars show the variability in all seasons compared to the mean.

The comparison of the diurnal tidal phase (Fig. 6a) shows agreement below $90 \mathrm{~km}$ for both seasons within the uncertainties. Above $90 \mathrm{~km}$ the phase difference increases with increasing altitudes and can be up to $\sim 12 \mathrm{~h}$ for the summer and $\sim 6 \mathrm{~h}$ for the winter. The diurnal meridional component shows similar agreement only up to $82 \mathrm{~km}$ and above the offset increases and reaches differences of $\sim 12 \mathrm{~h}$ during summer and winter.

\subsection{Correlation}

One goal of this study is to combine the two data sets to close gaps in the time series due to maintenance of one of the radars. To achieve that, it is necessary to find whether there is a general offset in the wind between the two systems. In order to generate a homogenous wind time series we intend to remove biases by defining one system as a reference.

Based on the estimated winds for both radars, the correlation coefficient $R^{2}$ is determined for different altitudes and times through the year. We apply two different approaches. 

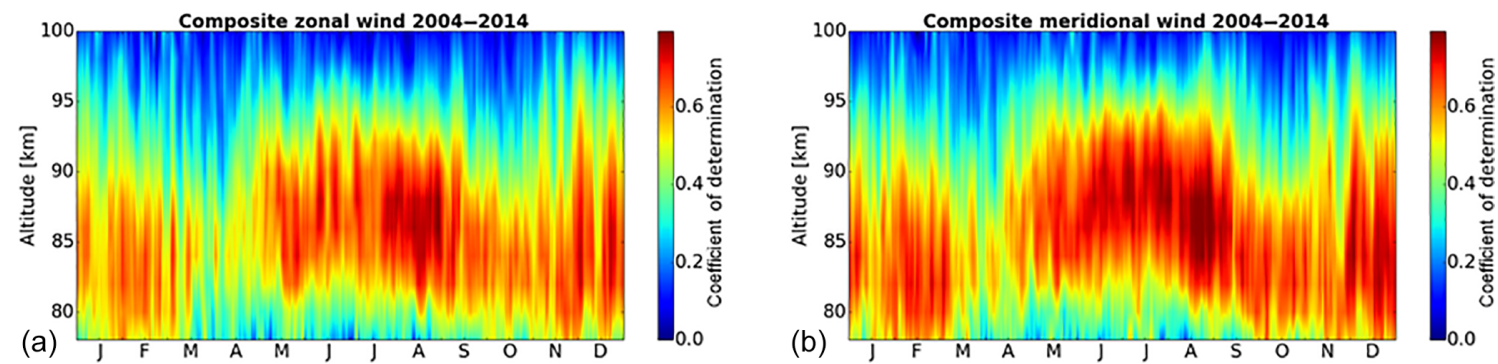

Figure 7. Composite of $R^{2}$ for the zonal (a) and meridional (b) component of the overlapped altitude of MF radar and meteor radar for the years 2004-2014. The data have been smoothed by using a 5-day running mean.

The first uses a 5-day running mean centered to the respective day. With this approach we estimate $R^{2}$ using hourly wind values over 5 days for each system. Figure 7 shows the resulting annual climatology for the zonal and meridional wind component. For the zonal wind component the highest correlation occurs during the summertime between 84 and $90 \mathrm{~km}$. This is the area in which the wind transition between the eastward and westward wind occurs. During the spring the correlation drops below 0.5 , which fits well with the occurrence of the wind transition during that time. Above $92 \mathrm{~km}$ the correlation drops below 0.5 and decreases further with increasing altitude. The same pattern is formed for the meridional wind component with decreasing correlation during the spring wind transition and above $94 \mathrm{~km}$.

The second approach aims to remove potential biases between the two data sets shown in Fig. 8. To find a possible linear relationship according to the least square fit method, one radar system needs to be defined as an independent and the other as a dependent variable. According to Hall et al. (2005), based on different measurement volumes, it is not possible to determine, a priori, one of the radar data sets as an independent variable. Several studies have shown good agreement between meteor radar and other instruments and models (e.g., Jacobi et al., 2009, Stober et al., 2012, McCormack et al., 2017). Therefore we use for our study the winds of the MR as a reference. Figure 8 shows a scatter plot for 80 , 86 , and $92 \mathrm{~km}$ for the zonal and meridional wind component of hourly wind values for the complete time period. These show a decreased correlation accompanied by an increased tilt of the scatter for increasing heights. The increased tilt is caused by the stronger winds of the MR, as shown in Fig. 3. The MF winds tend to systematically underestimate the winds compared to the MR at all altitudes. The colors of the scatter represent the counts of the compared hourly wind values of both systems at the same time step.

Table 2 shows $R^{2}$ for the hourly wind values for the years 2004-2014 without any smoothing for the following cases: all zonal wind values, all meridional wind values, only February zonal winds, and only June zonal winds. The mean correlation values are slightly higher for the zonal wind component $(0.55)$ than for the meridional wind $(0.50)$. These val- (a)
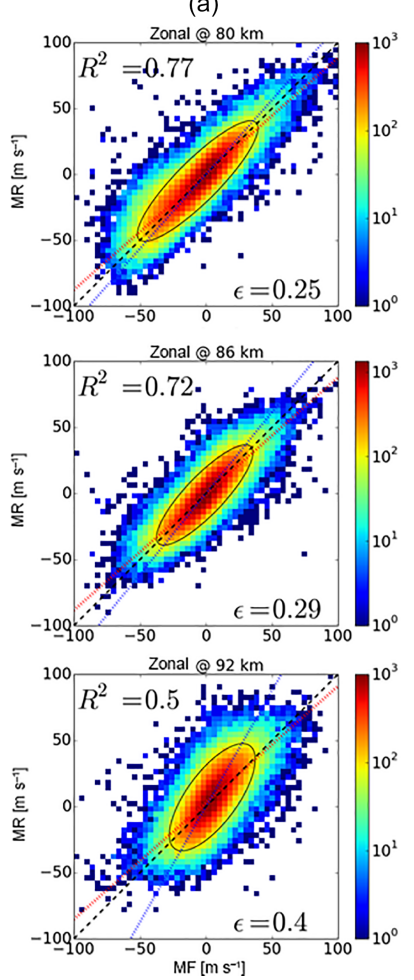

(b)
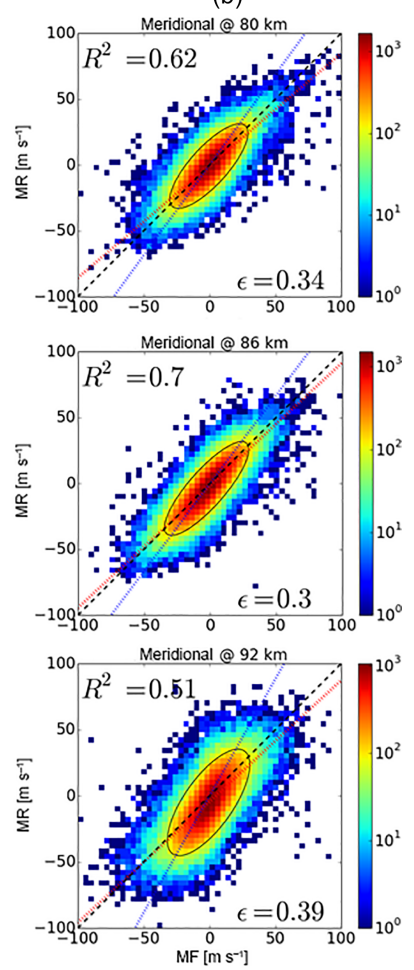

Figure 8. Scatter plots of MR versus MF zonal (a) and meridional (b) wind component for 80,86 , and $92 \mathrm{~km}$. The contour shows the number of wind values for 2004-2014. The black dashed line shows the line of equality. The red dashed line shows the least absolute deviation linear fit with MR as an independent variable, and the blue line is with MF as an independent variable. The ellipse within the contour plot is a criteria for the correlation of the two systems; $\epsilon$ is calculated by the width divided by the length of the ellipse.

ues decrease with increasing altitude, from 0.75/0.70 (zonal at 82 and meridional at $84 \mathrm{~km}$ ) to less than $0.4 / 0.4$ (zonal and meridional $>94 \mathrm{~km}$ ), which is in agreement with the results in Fig. 7. In addition to the altitudinal differences, we also observe seasonal differences with this approach. We found that for the zonal wind component, $R^{2}$ decreases for Febru- 


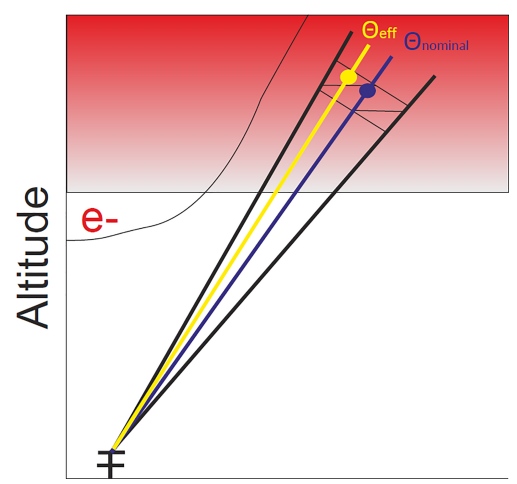

Figure 9. Schematic illustration of a tilted Saura beam (black) in a stratified atmosphere. The reddish area symbolizes an increasing electron density in the stratified medium. The observed tilted beam volume is not equally filled with larger electron density at the nearer-zenith beam edge. This results in a shifted beam pointing angle (yellow) relative to the main beam (blue).

ary from 0.79 to below 0.4 with increasing altitude. In contrast, however, $R^{2}$ for June increases between 78 and $88 \mathrm{~km}$ and decreases above $88 \mathrm{~km}$. The same pattern occurs for the meridional wind component.

The ellipse within the colored area in Fig. 8 is another measure for determining the correlation between the radars. The thinner the ellipse, the higher the correlation between the two data sets. The ratio $(\epsilon)$ between the width and the length of the ellipse indicates the quality of the ellipse. The values can vary between 0 and 1 with 0 as an ideal correlation independent of a possible offset between the data and 1 with no correlation between the two data sets. According to Table 2 the values for the zonal component vary between 0.25 and 0.3 below $86 \mathrm{~km}$ and increase with increasing height. The values for the meridional component are on average slightly higher.

\section{Discussion}

The aim of this study is the comparison of the obtained winds and tides from two different radar systems. On the one side we have the meteor radar and on the other side the mediumfrequency radar; the two systems are able to measure wind in an overlapping observation altitude between 78 and $100 \mathrm{~km}$. We compared data gathered between 2004 and 2014. Based on this comparison, we will present in the following section a method to combine both radar winds into a consistent and homogenous data set.

As a first step we analyzed the provided zonal and meridional wind components based on a running mean composite. The zonal wind structure in Fig. 3 exhibits the expected behavior with eastward-directed winds during the winter and a westward-dominated wind during the summer. By visual comparison of the annual climatology, which is computed from a 5-day running mean centered to the respective day, we obtain good agreement between the two systems below the altitude of $92 \mathrm{~km}$ for the zonal wind component, except during the wind transition period in spring. The presence of planetary waves in the wintertime can be seen in both radars. The main difference in the zonal wind component occurs above $92 \mathrm{~km}$ during the summer and shows for the meteor radar a strong eastward-directed wind pattern with mean values above $40 \mathrm{~m} \mathrm{~s}^{-1}$, which do not occur at the same amplitude for the medium-frequency radar with mean values around $10 \mathrm{~m} \mathrm{~s}^{-1}$.

The meridional wind component shows, as expected, lower amplitudes in both systems. Maximal wind values of $\pm 20 \mathrm{~m} \mathrm{~s}^{-1}$ can be seen in both systems. Below $92 \mathrm{~km}$ the wind pattern of both radars looks similar, according to Fig. 3, expect during the transition time in spring and autumn when the systems sometimes show opposite wind directions.

Moreover, we compare the wind directions based on hourly data. According to Fig. 4, below $92 \mathrm{~km}$ the directions are mainly identical and above $92 \mathrm{~km}$ discrepancies occur, which is shown in a displacement to the line of equality. This phenomenon occurs for the whole year and increases with increasing altitudes.

Especially for lower wind values, these wind differences can be attributed to the different observation volumes of the two systems with $\sim 400 \mathrm{~km}$ of diameter for the MR compared to $\sim 30 \mathrm{~km}$ of diameter for the MF at $90 \mathrm{~km}$ of altitude. In addition to that volume effect, Hall et al. (2005) found a systematic bias $(20 \%)$ in the meridional wind component by the MF radar for altitudes below $90 \mathrm{~km}$. They explained that the difference between the two systems occurs because the radars do not measure at the same altitude. The echoes of the MF radar measure in lower altitudes as expected. The reason for this is the group delay due to background ionization. Another reason could be that the signal the MF radar receives is purely due to the neutral wind (Hall et al., 2005). Manson et al. (2004) note that for the zonal wind component the difference is altitude dependent. They show good agreement during summer but with lower MF values (20$50 \%$ ) during the winter. Jacobi et al. (2009) further support these results. In agreement with their findings, we discovered an underestimation for the meridional wind below $92 \mathrm{~km}$ of approximately $10-60 \%$, which varies with season and altitude. The zonal component also shows lower wind values for the Saura MF in nearly every season and at every altitude. A reason to use the measured winds with Saura MF carefully above $92 \mathrm{~km}$ is due to E-region total reflection and the group retardation near midday (Reid, 2015). A study by McCormack et al. (2017), in which the Andenes MR was compared with the Navy Global Environmental Model (NAVGEM), a global spectral forecast model with a data assimilation algorithm, showed good agreement between their model and the MR measurements for the overlapping altitude range of each mean wind component. Furthermore a comparison between the VHF radar system MAARSY (Middle Atmosphere Alomar Radar System) and the MR at Andenes was done with 
Table 2. Coefficient of determination for the altitudes 78 to $100 \mathrm{~km}$ for the zonal and meridional wind component based on hourly wind values. February and June $R^{2}$ are for the zonal wind component, and $\epsilon$ describes the ratio between the length and the thickness of the scatter plot.

\begin{tabular}{lrrrrrr}
\hline Altitude & $R^{2}$ zonal & $R^{2}$ meridional & $R^{2}$ only Feb & $R^{2}$ only June & $\epsilon$ zonal & $\epsilon$ meridional \\
\hline 78 & 0.75 & 0.55 & 0.78 & 0.27 & 0.27 & 0.38 \\
80 & 0.77 & 0.62 & 0.79 & 0.38 & 0.25 & 0.34 \\
82 & 0.78 & 0.67 & 0.77 & 0.51 & 0.25 & 0.31 \\
84 & 0.77 & 0.70 & 0.74 & 0.61 & 0.26 & 0.30 \\
86 & 0.72 & 0.70 & 0.68 & 0.67 & 0.29 & 0.30 \\
88 & 0.66 & 0.67 & 0.61 & 0.66 & 0.32 & 0.31 \\
90 & 0.58 & 0.60 & 0.52 & 0.62 & 0.36 & 0.35 \\
92 & 0.50 & 0.51 & 0.45 & 0.54 & 0.40 & 0.39 \\
94 & 0.41 & 0.40 & 0.36 & 0.44 & 0.44 & 0.44 \\
96 & 0.30 & 0.29 & 0.27 & 0.30 & 0.48 & 0.49 \\
98 & 0.21 & 0.20 & 0.16 & 0.16 & 0.52 & 0.52 \\
100 & 0.14 & 0.12 & 0.12 & 0.09 & 0.54 & 0.53 \\
\hline Mean & 0.55 & 0.50 & 0.52 & 0.44 & 0.36 & 0.39 \\
\hline
\end{tabular}
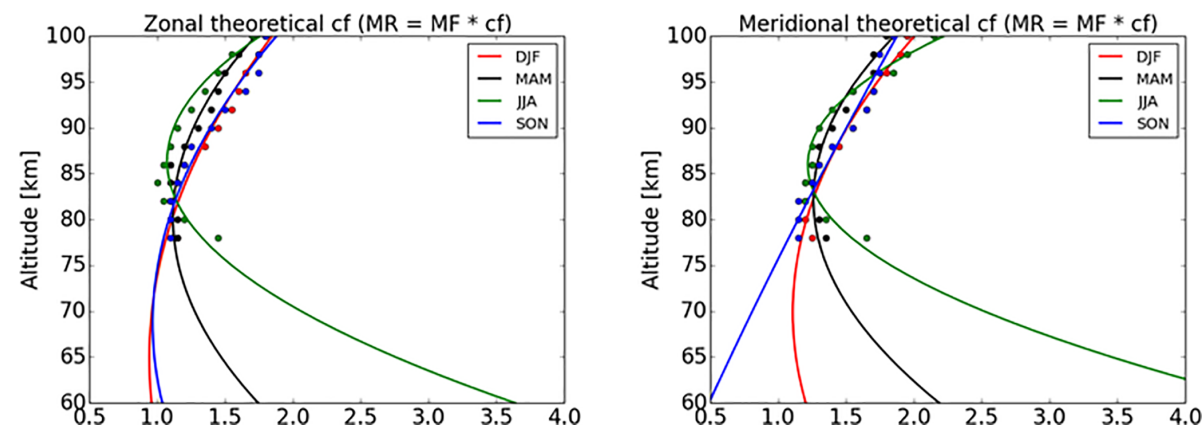

Figure 10. Theoretical seasonal correction factor for the zonal and meridional wind component of the MF radar. The points between 78 and $100 \mathrm{~km}$ are based on a comparison between the two radars according to Fig. 11. The fitted curves are polynomial functions of second degree according to Table 3 .

correlations for the zonal (0.78) and meridional (0.79) wind components that supports the quality of the MR winds (Stober et al., 2012).

Differences in the wind measurements between the MR and the MF radars occur for two reasons. First, under the assumption of a stratified mesosphere, which means that the mesosphere is homogenously filled with electron irregularities for every layer, the measured center of scatters in a tilted Saura beam (Fig. 9) is not necessarily in the middle of the beam volume (theta nominal). The measured center of backscatter is weighted by the electron density (theta eff) within the beam. In most cases the scattering center is weighted to lower zenith angles and therefore higher altitudes. This effect also plays an important role during strong electron events in the $\mathrm{D}$ and $\mathrm{E}$ regions. With higher altitudes this effect will increase due to broadening of the beam and can explain the differences between the MR radar and MF radar below $92 \mathrm{~km}$ in the wind amplitude and partially in the wind direction.
A second effect that influences the wind-derived measurements of the Saura radar is the scattering process. According to Singer (2003) and Singer (2007), sometimes there are differences of up to $10 \mathrm{~dB}$ between the main lobe of a vertical pointed narrow Doppler beam and the appropriate side lobes. However, the dynamic range of scattering in the D layer is about $35 \mathrm{~dB}$. This side lobe contamination may affect the Doppler measurement itself, as the spectra contain multiple or show smeared and asymmetric peaks, which are difficult to take into account with the momentum method. More information about the momentum method can be found in Strauch et al. (1984). Thus the analysis is not able to derive a reliable radial velocity for these altitudes. Another relevant effect above $90 \mathrm{~km}$ is the pointing of the beam itself, the range, and the Doppler measurement. The electron density reaches values of $10^{11} e-\mathrm{m}^{-3}$ (Bilitza and Renisch, 2007) that require a more complex analysis, including height retardation, similar to ionosondes. Further, the beam pointing is no longer given by the pointing geometry alone, but becomes more and more affected by wave refraction. Considering electron den- 

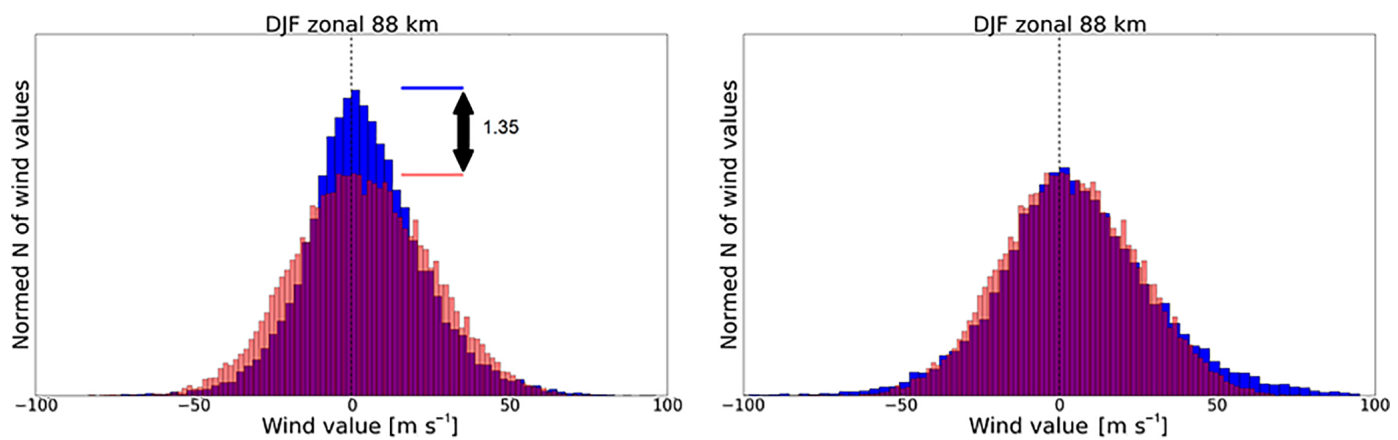

Figure 11. Comparison of sorted hourly wind values according to season, here DJF. MR (red) with values determined by the radar, MF (blue) with original values (left side), and values multiplied (right side) with a proportionate number according to the correction function of Table 3 to receive the same shape as the MR.

Table 3. Seasonal parameters used for a polynomial function to minimize the amplitude difference of the MF radar to the MR.

\begin{tabular}{lrrr}
\hline & a0 & a1 & a2 \\
\hline \multicolumn{4}{c}{ Zonal } \\
\hline DJF & 4.03 & -0.0952 & 0.000734 \\
MAM & 11.0 & -0.248 & 0.00155 \\
JJA & 28.6 & -0.637 & 0.00369 \\
SON & 5.38 & -0.128 & 0.000933 \\
\hline \multicolumn{4}{c}{ Meridional } \\
\hline DJF & 22.21 & -0.5078 & 0.0030875 \\
MAM & 31.67 & -0.7067 & 0.0041333 \\
JJA & 71.78 & -1.615 & 0.0092282 \\
SON & 23.44 & -0.5217 & 0.0030844 \\
\hline
\end{tabular}

sities close to $10^{11} e-\mathrm{m}^{-3}$ shows that the refractive index deviates significantly from $n=1$ at medium and high frequencies. Assuming a typical electron density profile, the refractive index $n$ is between 0.4 and 0.8 for altitudes above $92 \mathrm{~km}$ and the Saura frequency. This implies that the range and the Doppler measurements need to be corrected for the groupand phase-velocity effects.

It would be possible to improve the measurement of Saura by applying interferometry or imaging to account for the angular or range distribution of velocities; imaging Doppler interferometry (IDI; more information can be found in, e.g., Reid, 2015) could be a suitable approach for more reliable radial velocity measurements in the $\mathrm{D}$ layer, but in retrospect this adjustment cannot be done for the existing data. However, within this study we compare the winds of the MF and MR to find a statistical correction factor to construct a merged MR-MF time series for the complete available data set of both systems. For altitudes that are likely not affected by wave refraction, a correction factor is used to estimate an average pointing difference from the nominal beam pointing.
Based on the hourly wind values of both systems, we are able to determine a correlation for both wind components for every year by using a running mean boxcar. The shape of the correlation coefficient pattern (Fig. 7) shows the expected reasonable agreement below $92 \mathrm{~km}$ nearly for the whole year. The lower altitudes during summer and the wind transition time during spring show decreased correlations. The values of the meridional wind component are, according to these figures, higher than for the zonal component. The reason for the larger meridional values is an artifact due to the running boxcar method. According to Table 2 the values for the meridional wind are lower but the general pattern of the meridional figures is trustworthy. Manson et al. (2004) estimated a correlation $(R)$ between the Esrange MR and the Troms $\varnothing$ MF radars at a height of $88 \mathrm{~km}$ for winter (zonal) with 0.71 , winter (meridional) with 0.75 , summer (zonal) with 0.8 , and summer (meridional) with 0.83 . Our $R^{2}$ shows similar values for all cases (Table 2). The difference between Manson et al. (2004) and our findings can be explained by different lengths of the observation time and further by our hourly data compared to get the same shape for the histogram as the 2-hourly mean data of Manson et al. (2004).

The amplitude pattern of the tidal components (Fig. 5) is similar to the wind components with strong differences in the amplitude above $92 \mathrm{~km}$ for both components. The tidal amplitude of the MF radar does not grow with higher altitude due to no increasing winds with height. The semidiurnal tide is the main dominant tidal component in both systems, which fits with the results of Hoffmann et al. (2010). Yu et al. (2015) showed, however, increasing tidal amplitudes with increasing heights for several locations. The amplitudes in tidal modes vary with height, season, component, and location. Further studies investigated differences in the tidal components between MF and MR at Troms $\varnothing$ with the result that the surface topography influences the deposition of momentum flux, and therefore a tidal acceleration can be expected to vary with altitude over the observed area and with season (McIntyre, 1989, McLandress, 1998, Hall et al., 2005). In our case the surface topography does not play such an im- 

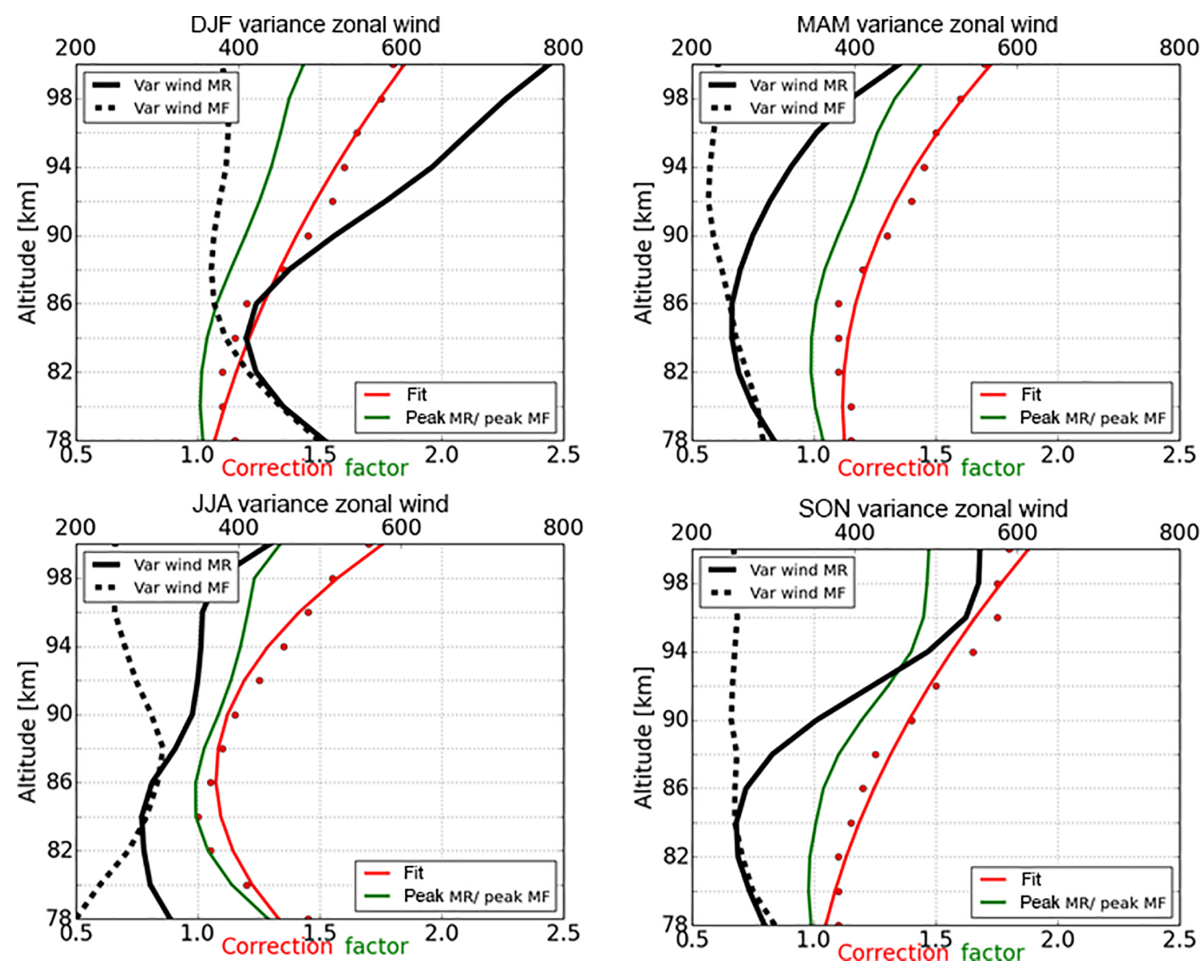

Figure 12. Seasonal plots to adjust the difference in the zonal wind value between the MF and the MR based on two different methods (colored). The correction factor is estimated by sorting the hourly winds according to their values and multiplying the correction factor (CF) on the MF value to get the same shape for the histogram as the MR (see Fig. 11). This is done for every altitude and season manually (red) and by dividing the maximum peak of MR by the maximum peak of MF (green). In black is the variance in the hourly winds for the corresponding season for MF (dashed) and MR (solid) based on hourly wind values over the whole time period for every altitude.
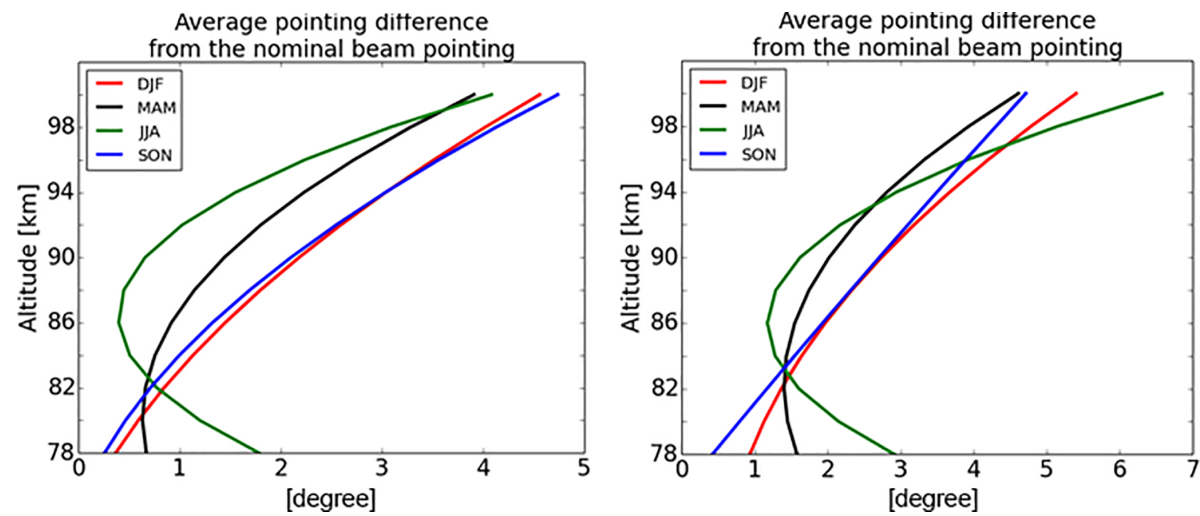

Figure 13. Theoretical average pointing difference from the nominal beam pointing of the Saura radar for DJF (red), MAM (black), JJA (green), and SON (blue) for the years 2004-2014. The left panel shows the zonal wind component, and the right panel shows the meridional wind component.

portant role because the two systems are only $20 \mathrm{~km}$ apart. By taking wind values within a $1 \mathrm{~h}$ bin, the influence of different measurement volumes of both systems and a strong change in the wind to the previous and next time bin can almost be neglected. Along with the altitudinal differences in the tidal amplitude, a distinct difference also occurs between the semidiurnal and diurnal components; the correla- tion between the two systems for the $12 \mathrm{~h}$ tide is higher than for the $24 \mathrm{~h}$ tide with maximal values for the zonal case of $\sim 0.6$ compared to $\sim 0.4$ (not shown here). These values decrease with increasing altitudes. In addition to the amplitudes of the tidal components we compared the phases with a similar result. Below $88 \mathrm{~km}$ for the diurnal and $94 \mathrm{~km}$ for the semidiurnal component, the phases of both systems are in 

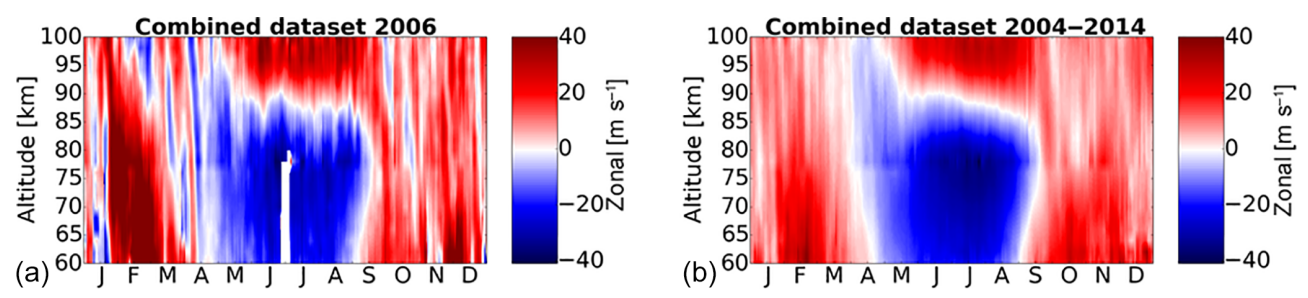

Figure 14. Zonal wind component for the year 2006 (a) and the composite for the years 2004-2014 (b). Below $78 \mathrm{~km}$ the data are based on the MF system, and above $92 \mathrm{~km}$ they are based on the MR radar. The overlapping area is based on the MR and the gaps within this area are filled with weighted MF radar data according to the correction functions in Table 3. The white areas during June 2006 are missing values.

good agreement within the uncertainties, but the difference increases with increasing heights up to a time delay of $12 \mathrm{~h}$ for the diurnal phases.

Due to the mentioned reasons and under the assumption that the derived tidal components of the MR are correct, we recommend that the tidal components based on the MF should not be used for tidal studies above $92 \mathrm{~km}$.

On the basis of these findings we propose MR wind measurements as primary wind for the overlapping coverage and filling existing gaps with weighted MF winds with respect to each wind component, altitude, and seasonal appearance. By comparing the amplitude wind differences between the two systems, we estimated theoretical correction factors (CFs) that fit, in most cases, to a polynomial function of second degree. Table 3 shows the parameters for the polynomials for each season and wind component. By using these parameters we show in Fig. 10 a theoretical profile of the CF down to $60 \mathrm{~km}$ for both wind components. The points between 78 and $100 \mathrm{~km}$ show estimated CFs based on hourly wind amplitudes without any smoothing but with respect to seasons. The estimation of these CFs is done by comparing these data for every altitude and according to their shape and multiplying the MF radar data with an associated CF (Fig. 11). Figure 10 shows good agreement between the polynomial function and the estimated correction factors for all seasons. The highest theoretical CF below the overlapping area can be found during the summer. It should also be briefly mentioned that below and above the overlapping area these functions need further investigation because polynomials with a higher degree also fit within the overlapping area; beyond the overlapping area, where the $\mathrm{CF}$ is extrapolated, strong differences appear.

Figure 12 shows vertical profiles of the observed difference factors and our empirical correction factors. The green line shows the correction factors based on dividing the peak of MR by the peak of MF, and the red line and red dots are CFs and correction functions of Fig. 10. Both curves show a similar pattern. The black curves are the seasonal wind variances for MR (solid) and MF (dashed), which increase in the case of the MR with increasing heights. The variance curve of the MR fits well in shape with the two CF lines. This illustrates that one reason for the shape of the correction function is the growing wind values of the MR compared to the MF.
With the use of CF we show in Fig. 14 two examples for a combined zonal wind data set of both systems with altitude coverage between 60 and $100 \mathrm{~km}$. They are based on a 5-day running mean for the year 2006 (Fig. 14a) and as a composite for the years 2004-2014 (Fig. 14b). Below $78 \mathrm{~km}$ the combined data set of the MF system is shown without the correction factor and above $92 \mathrm{~km}$ with only the meteor radar data. In the area in between we take mainly the MR data and fill gaps with the Saura radar data winds by applying the correction factors in Table 3. In general this leads to good results for both figures. Only the transition between $78 \mathrm{~km}$ and the altitudes below, at which the MF winds without any weighted function are connected, tend to still contain a small offset between the MF and MR winds.

Based on the correction factor we estimate an average pointing difference from the nominal beam pointing of the Saura beam, which can be seen in Fig. 13. Theoretically, by using a modified value of the Saura zenith pointing angle in the analysis, the MF and MR winds would agree better.

\section{Conclusions}

In this study we have compared the winds of the Saura MF and Andenes MR by applying a DBS wind analysis for both systems. Data from 11 years have been studied with the objective of obtaining a vertical wind profile between approximately 60 and $110 \mathrm{~km}$. To acheive this, biases between the two systems were determined, mainly to remove systemspecific differences, such as scattering processes, technical setup, and frequencies. Inside the overlapping altitude in the range from 78 to $100 \mathrm{~km}$, the highest agreement (zonal and meridional $R^{2}=0.78 / 0.70$ ) of the two wind components is between 78 and $94 \mathrm{~km}$, except during the transition time (spring) and during the summer below $82 \mathrm{~km}$. Above $92 \mathrm{~km}$ the correlation decreases with increasing altitude. It is clear that there is no perfect correlation between the two radars, especially on shorter timescales, which is due to fundamental differences in the systems, e.g., different measurement volume, frequency, and scattering processes. We compared the derived tidal components between the two radars. The amplitudes and the phases of the diurnal and semidiurnal tides from both sets of measurements are in agreement be- 
low $90 \mathrm{~km}$. With increasing altitudes above $90 \mathrm{~km}$, the mean semidiurnal phases are in agreement within $\pm 6 \mathrm{~h}$ and the diurnal phases are within $\pm 12 \mathrm{~h}$. The use of tidal phases at these heights should therefore be taken with caution. The best agreement occurs during the winter period below $90 \mathrm{~km}$ for the semidiurnal tide. With increasing altitudes the agreement decreases because the phase and the amplitude of the MFbased tides remain almost constant with increasing altitude. This is not supported by the MR observations, which show a clear phase propagation with altitude and increasing altitudes. Based on our findings we provide a correction function for every season to minimize differences in wind amplitudes between the two systems. These correction functions fit to a polynomial function of second order, but should only be used for the altitudes at which both systems are able to obtain winds. Extrapolating the correction function beyond the overlapping area can cause problems and needs further investigation. By combining the MR and the weighted MF data set, we are able to construct a continuous data set with altitude coverage from 60 to $110 \mathrm{~km}$ over 11 years, which can be used for further studies.

Data availability. The radar data are available upon request from Gunter Stober (stober@iap-kborn.de).

Competing interests. The authors declare that they have no conflict of interest.

Acknowledgements. This work was partly supported by the WaTiLa project (SAW-2015-IAP-1 383) and partly by the Deutsche Forschungsgemeinschaft (DFG, German Research Foundation; project no. LU1174, PACOG). We acknowledge the technical support of the IAP technicians and are thankful for the discussions with Peter Hoffmann, Toralf Renkwitz, and Carsten Schult.

The topical editor, Keisuke Hosokawa, thanks Chris Meek and one anonymous referee for help in evaluating this paper.

\section{References}

Andrews, D. G., Holton, J. R., and Leovy, C. B.: Middle atmosphere dynamics, Academic Press, New York, NY, USA, 489 pp., 1987.

Aster, R. C. and Borchers, B. T. C.: Parameter Estimation and Inverse Problems, Academic Press, Elsevier, 2 Edn., 2013.

Bilitza, D. and Renisch, B.: International Reference Ionosphere 2007: Improvements and new parameters, Adv. Space Res., 42, 599-609, https://doi.org/10.1016/j.asr.2007.07.048, 2007.

Briggs, B. H.: The analysis of spaced sensor records by correlation techniques, Handbook for MAP, 13, 166-186, 1984.

Browning, K. and Wexler, R.: The determination of kinematic properties of a wind field using doppler radar, J. Appl. Meteorol., 7, 105-113, 1968.

Chau, J. L., Röttger, J., and Rapp, M.: PMSE strength during enhanced $\mathrm{D}$ region electron densities: Faraday rotation and absorp- tion effects at VHF frequencies, J. Atmos. Sol.-Terr. Phys., 118, 113-118, https://doi.org/10.1016/j.jastp.2013.06.015, 2014.

Fritts, D. C., Iimura, H., Lieberman, R., Janches, D., and Singer, W.: A conjugate study of mean winds and planetary waves employing enhanced meteor radars at Rio Grande, Argentina $\left(53.8^{\circ} \mathrm{S}\right)$ and Juliusruh, Germany (54.6 $\left.{ }^{\circ} \mathrm{N}\right)$, J. Geophys. Res.-Atmos., 117, D05117, https://doi.org/10.1029/2011JD016305, 2012.

Hall, C., Aso, T., Tsutsumi, M., Nozawa, S., Meek, C., and Manson, A.: Comparison of meteor and medium frequency radar kilometer scale MLT dynamics at $70^{\circ}$ N, J. Atmos. Sol.-Terr. Phys., 68, 309-316, https://doi.org/10.1016/j.jastp.2005.03.025, 2006.

Hall, C. M., Aso, T., Tsutsumi, M., Nozawa, S., Manson, A. H., and Meek, C. E.: A comparison of mesosphere and lower thermosphere neutral winds as determined by meteor and medium-frequency radar at $70^{\circ} \mathrm{N}$, Radio Sci., 40, RS4001, https://doi.org/10.1029/2004RS003102, 2005.

Hocking, W., Thayaparan, T., and Franke, S.: Method for statistical comparison of geophysical data by multiple instruments which have differing accuracies, Adv. Space Res., 27, 10891098, https://doi.org/10.1016/S0273-1177(01)00143-0, $2001 \mathrm{~b}$.

Hocking, W. K. and Thayaparan, T.: Simultaneous and colocated observation of winds and tides by $\mathrm{MF}$ and meteor radars over London, Canada (43 N, 81 ${ }^{\circ} \mathrm{W}$ ), during 1994-1996, Radio Sci., 32, 833-865, https://doi.org/10.1029/96RS03467, 1997.

Hocking, W. K., Fuller, B., and Vandepeer, B.: Realtime determination of meteor-related parameters utilizing modern digital technology, J. Atmos. Sol.-Terr. Phys., 69, 155-169, https://doi.org/10.1016/S1364-6826(00)00138-3, 2001a.

Hoffmann, P., Becker, E., Singer, W., and Placke, M.: Seasonal variation of mesospheric waves at northern middle and high latitudes, J. Atmos. Sol.-Terr. Phys., 72, 1068-1079, https://doi.org/10.1016/j.jastp.2010.07.002, 2010.

Hooper, D. A., Nash, J., Oakley, T., and Turp, M.: Validation of a new signal processing scheme for the MST radar at Aberystwyth, Ann. Geophys., 26, 3253-3268, https://doi.org/10.5194/angeo26-3253-2008, 2007.

Iimura, H., Fritts, D. C., Janches, D., Singer, W., and Mitchell, N. J.: Interhemispheric structure and variability of the 5-day planetary wave from meteor radar wind measurements, Ann. Geophys., 33, 1349-1359, https://doi.org/10.5194/angeo-33-1349-2015, 2015.

Jacobi, C., Arras, C., Kürschner, D., Singer, W., Hoffmann, P., and Keuer, D.: Comparison of mesopause region meteor radar winds, medium frequency radar winds and low frequency drifts over Germany, Adv. Space Res., 43, 247-252, https://doi.org/10.1016/j.asr.2008.05.009, 2009.

Jones, J., Webster, A. R., and Hocking, W. K.: An improved interferometer design for use with meteor radars, Radio Sci., 33, 55-65, https://doi.org/10.1029/97RS03050, 1998.

Manson, A. H., Meek, C. E., Hall, C. M., Nozawa, S., Mitchell, N. J., Pancheva, D., Singer, W., and Hoffmann, P.: Mesopause dynamics from the scandinavian triangle of radars within the PSMOS-DATAR Project, Ann. Geophys., 22, 367-386, https://doi.org/10.5194/angeo-22-367-2004, 2004.

McCormack, J., Hoppel, K., Kuhl, D., de Wit, R., Stober, G., Espy, P., Baker, N., Brown, P., Fritts, D., Jacobi, C., Janches, D., Mitchell, N., Ruston, B., Swadley, S., Viner, K., Whitcomb, T., and Hibbins, R.: Comparison of mesospheric winds from a high-altitude meteorological analysis system and meteor radar observations during the boreal winters of 2009- 
2010 and 201-2013, J. Atmos. Sol.-Terr. Phys., 154, 132-166, https://doi.org/10.1016/j.jastp.2016.12.007, 2017.

McIntyre, M. E.: On dynamics and transport near the polar mesopause in summer, J. Geophysical Res.-Atmos., 94, 1461714628, https://doi.org/10.1029/JD094iD12p14617, 1989.

McLandress, C.: On the importance of gravity waves in the middle atmosphere and their parameterization in general circulation models, J. Atmos. Sol.-Terr.1 Phys., 60, 1357-1383, https://doi.org/10.1016/S1364-6826(98)00061-3, 1998.

Rapp, M., Strelnikova, I., Latteck, R., Hoffmann, P., Hoppe, U.P., Häggström, I., and Rietveld, M. T.: Polar mesosphere summer echoes (PMSE) studied at Bragg wavelengths of $2.8 \mathrm{~m}$, $67 \mathrm{~cm}$, and $16 \mathrm{~cm}$, J. Atmos. Sol.-Terr. Phys., 70, 947-961, https://doi.org/10.1016/j.jastp.2007.11.005, 2008.

Reid, I. M.: MF and HF radar techniques for investigating the dynamics and structure of the 50 to $110 \mathrm{~km}$ height region: a review, Prog. Earth Planet. Sci., 2, 33 pp., https://doi.org/10.1186/s40645-015-0060-7, 2015.

Singer, W., Latteck, R., Holdsworth, D. A., and Kristiansen, T. (Eds.): A new narrow beam MF Radar at $3 \mathrm{MHz}$ for studies of the high-latitude middle atmosphere: System description and first results, Proceedings of the 10th international workshop on technical and scientific aspects of MST radar, 2003.

Singer, W., Latteck, R., and Holdsworth, D.: A new narrow beam Doppler radar at $3 \mathrm{MHz}$ for studies of the high-latitude middle atmosphere, Adv. Space Res., 41, 1488-1494, 2008.

Sommer, S., Stober, G., and Chau, J. L.: On the angular dependence and scattering model of polar mesospheric summer echoes at VHF, J. Geophys. Res.-Atmos., 121, 278-288, https://doi.org/10.1002/2015JD023518, 2016.

Stober, G. and Chau, J. L.: A multistatic and multifrequency novel approach for specular meteor radars to improve wind measurements in the MLT region, Radio Sci., 50, 431-442, https://doi.org/10.1002/2014RS005591, 2015.
Stober, G., Latteck, R., Rapp, M., Singer, W., and Zecha, M.: MAARSY - the new MST radar on Andøya: first results of spaced antenna and Doppler measurements of atmospheric winds in the troposphere and mesosphere using a partial array, Adv. Radio Sci., 10, 291-298, https://doi.org/10.5194/ars-10-291-2012, 2012.

Stober, G., Matthias, V., Jacobi, C., Wilhelm, S., Höffner, J., and Chau, J. L.: Exceptionally strong summer-like zonal wind reversal in the upper mesosphere during winter 2015/16, Ann. Geophys., 35, 711-720, https://doi.org/10.5194/angeo-35-711-2017, 2017.

Strauch, R., Merritt, D., Moran, K., Earnshaw, K., and Van De Kamp, D.: The Colorado Wind-Profiling Network, J. Atmos. Ocean. Technol., 1, 37-49, 1984.

Suzuki, H., Nakamura, T., Ejiri, M. K., Ogawa, T., Tsutsumi, M., Abo, M., Kawahara, T. D., Tomikawa, Y., Yukimatu, A. S., and Sato, N.: Simultaneous PMC and PMSE observations with a ground-based lidar and SuperDARN HF radar at Syowa Station, Antarctica, Ann. Geophys., 31, 1793-1803, https://doi.org/10.5194/angeo-31-1793-2013, 2013.

Valentic, T. A., Avery, J. P., Avery, S. K., and Vincent, R. A.: A comparison of winds measured by meteor radar systems and an MF radar at Buckland Park, Radio Sci., 32, 867-874, https://doi.org/10.1029/96RS03308, 1997.

Vierinen, J., Chau, J. L., Pfeffer, N., Clahsen, M., and Stober, G.: Coded continuous wave meteor radar, Atmos. Meas. Tech., 9, 829-839, https://doi.org/10.5194/amt-9-829-2016, 2016.

Waldteufel, P. and Corbin, H.: On the Analysis of Single-Doppler Radar Data, J. Appl. Meteorol., 18, 532-542, 1978.

Yu, Y., Wan, W., Ren, Z., Xiong, B., Zhang, Y., Hu, L., Ning, B., and Liu, L.: Seasonal variations of MLT tides revealed by a meteor radar chain based on Hough mode decomposition, J. Geophys. Res.-Space, 120, 7030-7048, https://doi.org/10.1002/2015JA021276, 2015. 